\title{
Réponse de poulets normaux et nains (gène $d w$ ) à une privation d'aliment en phase de croissance
}

\author{
A. EL ATTAR (*), A. BORDAS et P. MERAT \\ I.N.R.A., Laboratoire de Génétique factorielle, \\ Centre de Recherches zootechniques; $F 78350$ Jouy-en-Josas
}

\begin{abstract}
Résumé
Les effets d'une privation d'aliment (eau ad libitum) pendant 72 heures à 8 semaines d'âge ont été examinés sur des poulets nains (mâles $d w d w$, femelles $d w$ ) et de taille normale (mâles $D w^{+} d w$, femelles $\mathrm{Dw}^{+}$) de même origine : femelles en cages individuelles, oiseaux des deux sexes au sol. Dans ce dernier cas, un lot non soumis au jeûne servait de témoin pour la reprise de poids après la privation d'aliment. Sur un autre échantillon de femelles, à partir de 11 semaines, la composition corporelle était comparée entre génotypes avec ou sans jeûne et une semaine après.

Dans l'ensemble, les effets du jeûne sur les poulets nains et sur ceux de taille normale diffèrent peu. Les " nains " perdent légèrement moins de poids en \% pendant le jeûne que les normaux, mais au sol seulement. Les différences entre génotypes concernant la température corporelle, le glucose ou l'acide urique plasmatique ne sont pas accusées par le jeûne. La reprise de poids relative durant les deux semaines consécutives est au total inférieure pour les animaux nains; elle est décalée dans le temps pour ce génotype et un peu supérieure la seconde semaine au sol. La première semaine en cages, l'indice de consommation n'est pas meilleur pour les poulettes normales que pour les naines; la remontée du pourcentage de lipides abdominaux après le jeûne à 11 semaines est plus précoce pour ce dernier génotype. Une augmentation de l'hématocrite des femelles dw pendant et après la privation d'aliment solide suggère une modification du bilan hydrique.
\end{abstract}

Mots clés : volailles, nanisme, jeûne.

\section{Summary}

\section{Response of normal and dwarf (dw gene) chicks to starvation during the growth} period.

The effects of starvation (water ad libitum) for 72 hours at 8 weeks of age have been studied in dwarf chicks (dwdw males and dw females) and normal-sized chicks of the same origin ( $D w^{+} \mathrm{dw}$ males, $\mathrm{Dw}^{+}$females). Only females were put into individual cages ; both sexes were kept in floor pens. In the latter case, body weight gain after the starvation period was compared to that of a control group without starvation. In another sample of females, the body composition of the two genotypes was compared, with or without starvation, at 11 weeks of age and 1 week later.

The effects of starvation were not very different in dwarf and normal birds. In floor pens only, relative body weight loss during starvation was slightly less in dwarfs than in normals. Genotypic differences in body temperature, plasma glucose and uric acid were not significantly altered by starvation. The recovery of relative body weight during the 11 days after starvation was less, on the whole, for dwarf birds ; they recovered body weight later and their recovery rate was higher during the second week in floor pens. During the first week after starvation in cages, the food efficiency of normal pullets was not better than that of the dwarfs ; the percentage of abdominal fat after the end of starvation at 11 weeks increased earlier in dw birds. An increase in the hematocrit of dw females during and after starvation suggested a modification in the water balance during that time.

Key words : poultry, dwarfism, starvation.

$\left({ }^{*}\right)$ Adresse permanente : Departement de Production animale, Faculté d'Agriculture, Université d'Aïn-Shams, Le Caire, Egypte. 


\section{Introduction}

Si les effets d'une privation d'aliment chez les oiseaux sont bien connus (voir BELL et FREEMAN, 1971), par contre on a peu recherché des différences entre génotypes en réponse à un jeûne. KAWAHARA (1962) compare des poussins d'un jour de plusieurs races pour leur survie en l'absence d'aliment : les poussins Plymouth Rock barrés survivent plus longtemps que des Leghorn. SIMON (1972) étudie l'influence du gène de nanisme dw sur la croissance compensatrice de poulets en réponse à un jeûne périodique. Les poussins de taille normale s'adaptent à l'alimentation discontinue en surconsommant pendant les phases de réplétion, les nains ne le font pas. UENO et al., (1977), sur des coqs de 35 semaines d'âge, observent une survie au jeûne, d'animaux Leghorn nains (dw), plus prolongée que celle de Leghorn de taille normale ou de Fayoumi. ZEIN-EL-DEIN et al., (1982), trouvent, lors d'un jeûne de 48 heures à l'âge de 8 ou 9 semaines, une perte relative de poids corporel légèrement mais non significativement plus faible pour des poulets " cou nu » que pour ceux à plumage normal.

Nous avons voulu réaliser une comparaison similaire à celle faite par ces derniers auteurs, relativement aux génotypes « taille normale " (gène $\mathrm{Dw}^{+}$) et " nanisme lié au sexe " (allèle dw). Il s'agit donc des effets d'un jeûne unique et non, comme dans l'étude de SIMON (1972), du résultat de diètes hydriques périodiquement répétées.

\section{Matériel et méthodes}

\section{A. Animaux et schéma expérimental}

Trente six femelles naines (dw) issues d'une souche expérimentale mi-lourde ont été inséminées artificiellement avec le sperme mélangé de 7 mâles hétérozygotes $D w+d w$, tous frères. Les descendants, au nombre de 390 , comprenaient des poussins « normaux 》 (mâles $D w^{+} d w$, femelles $D w^{+}$) et « nains » (mâles dwdw, femelles dw). La mère seule étant identifiée, ces poussins étaient bagués à l'éclosion et élevés sur litière jusqu'à la fin de la $3^{\text {e }}$ semaine. A cet âge, et pour des raisons matérielles, les animaux étaient répartis en trois groupes traités chacun de façon distincte :

$1-56$ femelles $\left(28 \mathrm{Dw}^{+}, 28 \mathrm{dw}\right)$ étaient choisies par familles et mises en cages individuelles permettant le contrôle de la consommation alimentaire. A 8 semaines d'âge, ces femelles étaient soumises à une diète hydrique (eau ad libitum), à partir de 17 heures le soir et pendant 72 heures, puis réalimentées ad libitum.

2 - 52 autres femelles étaient placées dans le même local que les précédentes, mais dans une batterie collective. A 11 semaines d'âge, elles étaient à leur tour soumises à un jeûne suivant une procédure identique à celle décrite ci-dessus.

3 - Le reste des femelles et les mâles étaient répartis au hasard, à l'intérieur de chaque famille de mère, en deux groupes élevés au sol dans deux loges identiques. A 8 semaines d'âge, l'un de ces deux groupes était soumis, en même temps que les femelles en cages indi- . viduelles, à un jeûne hydrique de même durée, l'autre groupe servant de témoin. 


\section{B. Conditions d'élevage}

Durant la première semaine, les poussins étaient élevés à $34^{\circ} \mathrm{C}$ sous éleveuse, puis la température était abaissée graduellement jusqu'à $28^{\circ} \mathrm{C}$ environ à la fin de la $3^{\mathrm{e}}$ semaine. Elle était maintenue, de la $4^{e}$ à la $10^{e}$ semaine, autour de $25^{\circ} \mathrm{C}$.

Pendant les trois premières semaines, les poussins étaient soumis à un éclairage continu. Ensuite, et jusqu'à la fin de l'expérience, les poussins élevés sur litières recevaient la lumière naturelle ; ceux élevés en cages individuelles ou batterie étaient éclairés artificiellement 10 heures par jour.

La ration, donnée ad libitum comme l'eau, à l'exception de la période du jeûne, était sous forme de farine et contenait $18 \%$ de protéines totales et $2820 \mathrm{Kcal} / \mathrm{Kg}$ d'énergie métabolisable, de l'éclosion à la fin de l'expérience.

La mortalité était de 7,1\% au total, déduction faite d'éliminations correspondant à des erreurs de sexage ou à l'absence de l'une des mesures. Elle ne différait pas selon le traitement.

\section{Identification des génotypes et mesures}

L'identification des génotypes (« normaux » et « nains ») était faite à 9 semaines d'âge par la longueur du tarse.

Les mesures prises dans chaque groupe étaient les suivantes :

\section{Au sol}

- Les animaux au sol étaient tous pesés à l'âge de 8 semaines, le matin du premier jour de jeûne, puis avant la fin de ce dernier pour ceux privés d'aliment. La perte de poids (absolue et en $\%$ du poids initial) en était déduite. Les poids à 9 et 10 semaines étaient mesurés pour tous les individus, permettant d'obtenir la reprise de poids après réalimentation, en valeur absolue et en \%. Tous les poids étaient enregistrés le matin, entre $9 \mathrm{~h}$ et $10 \mathrm{~h} 30$ environ, l'aliment ayant été retiré la veille au soir, vers 17 heures. La température rectale était mesurée avant le retrait d'aliment puis peu avant la fin du jeûne, chaque fois en fin d'après-midi, par une sonde reliée à un thermocouple (marque Ouvrard, Vilers \& Guilux).

\section{En cages individuelles}

- En cages individuelles, les mêmes variables étaient enregistrées. En outre, la consommation alimentaire par animal était notée pour les périodes où le gain de poids était mesuré. Pour les mêmes animaux, en début et en fin de jeûne, le taux plasmatique de glucose et d'acide urique était estimé par un analyseur semi-automatique Beckman.

Sur un échantillon de 12 couples de demi-sœurs ou sœurs, l'une naine, l'autre normale, parmi celles élevées en cages individuelles, les feces étaient récoltés sur 48 heures, à partir de la $16^{\mathrm{e}}$ heure de jeûne, puis une semaine après, pour en déterminer la quantité absolue et le pourcentage de matière sèche.

\section{Mesures sur carcasse}

- 8 couples de sœurs ou demi-sœurs, l'une normale, l'autre naine, en cages individuelles, étaient abattus à 11 semaines et servaient de témoin. Au même âge, mais après un 
jeûne de 72 heures, 10 couples de demi-sœurs ou sœurs (l'une $\mathrm{Dw}^{+}$, l'autre $\mathrm{dw}$ ) en batterie collective étaient également abattus. Une semaine plus tard, il en était de même pour dix couples similaires prélevés dans la même batterie collective. Sur tous ces animaux étaient déterminés le poids corporel avant et après abattage et saignée, la graisse abdominale (principalement autour du gésier, jabot, proventricule, bourse de Fabricius : RICARD et ROUVIER, 1976), le foie et sa teneur en matière sèche. L'hématocrite était noté à partir du sang prélevé lors de la saignée. Les poulettes élevées en batterie collective ont servi exclusivement à ces mesures.

\section{Analyse statistique}

- Les analyses de variance ou tests $t$ sont faits séparément à l'intérieur de chaque groupe élevé de façon différente (sol, cages individuelles). Le lot " témoin " élevé au sol n'est utilisé que pour comparer la reprise de poids après la période correspondant au jeûne.

\section{Résultats et discussion}

Le tableau 1 donne, pour les poulets élevés soit au sol, soit en cages individuelles, les valeurs moyennes pour les critères mesurés avant et après jeûne. En complément, le tableau 2 présente, pour les animaux élevés au sol, une analyse de variance avec les facteurs contrôlés " génotype » et « sexe " pour la perte de poids pendant le jeûne.

$\mathrm{Au}$ tableau 3 figurent, par génotype, dans chaque groupe expérimental, les valeurs moyennes pour la reprise de poids relative et l'indice de consommation pendant les 11 jours suivant le jeûne, ainsi que les tests de comparaison des moyennes entre génotypes.

Quant aux poids moyens de feces récoltées sur 48 heures lors du jeûne, ils sont respectivement de 4,4 $\mathrm{g}$ et $2,3 \mathrm{~g}$ pour les génotypes $\mathrm{Dw}^{+}$et $\mathrm{dw}(\mathrm{P}<0,001)$. Les valeurs une semaine après la reprise d'aliment sont respectivement 40,4 et $27,3 \mathrm{~g}$ pour les mêmes génotypes $(P<0,001)$. Aucune différence significative n'apparaît entre génotypes, dans les deux périodes, pour la teneur en eau des feces.

Le tableau 4 compare, pour chaque génotype, les critères de composition corporelle et l'hématocrite, à la fin d'un jeûne de 72 heures, une semaine après ou en l'absence de jeûne, sur l'échantillon et suivant les modalités décrites plus haut.

\section{A. Critères de réponse au jeûne}

La perte de poids relative (en $\%$ du poids corporel initial) n'est pas différente pour les poulettes "normales" et "naines" en cages individuelles (tableau 1). Au sol, cette perte est de l'ordre de $1 \%$ plus faible chez les animaux nains. A l'intérieur de chaque sexe, la différence entre génotypes s'approche du seuil de $5 \%$ de signification ; au total, elle se révèle significative à partir de l'analyse de variance du tableau 2. La différence du résultat, suivant que les poulets sont élevés au sol ou en cages, n'est pas expliquée. Par contre, des poules Leghorn adultes naines en ponte, soumises, en cages, à un jeûne analogue, perdent relativement plus de poids que leurs demi-sœurs normales (ROMBAUTs et al., sous presse). D'autre part, au sol, où la comparaison était possible, les femelles perdent un peu plus de poids en valeur relative que les mâles. La perte en \% est plus élevée chez les oiseaux en cages individuelles que pour ceux au sol, ce qui reste également inexpliqué. 


\section{TABleAU 1}

Poulets normaux et nains : réponse à 72 h de jeûne à 8 semaines d'âge.

Normal and dwarf chicks : response to 72 h starvation at 8 weeks of age.

\begin{tabular}{|c|c|c|c|c|c|c|c|}
\hline \multirow{3}{*}{ Caractère } & \multirow{3}{*}{ Sexe } & \multicolumn{6}{|c|}{ Valeurs moyennes et signification } \\
\hline & & \multicolumn{3}{|c|}{$\begin{array}{c}\text { Elevage au sol } \\
\text { (lot soumis au jeûne) }\end{array}$} & \multicolumn{3}{|c|}{ Cages individuelles } \\
\hline & & Normaux & Nains & $\begin{array}{l}\text { Significa- } \\
\text { tion de la } \\
\text { différence } \\
\text { (test } t)\end{array}$ & Normaux & Nains & $\begin{array}{l}\text { Significa- } \\
\text { tion de la } \\
\text { différence } \\
\text { (test } \mathrm{t} \text { ) }\end{array}$ \\
\hline Nombre d'animaux. . & $\sigma^{0}$ & 38 & 40 & & - & - & \\
\hline & Q & 18 & 30 & & 28 & 28 & \\
\hline $\begin{array}{l}\text { Poids à } 8 \text { sem. } \ldots \ldots \\
\text { avant jeûne }(g) \ldots \ldots\end{array}$ & ơ & $\begin{array}{l}848 \\
751\end{array}$ & $\begin{array}{l}616 \\
545\end{array}$ & $\begin{array}{l}* * * \\
* * *\end{array}$ & 818 & 606 & $* * *$ \\
\hline $\begin{array}{l}\text { Perte de poids rela- } \\
\text { tive }(\% \text { du poids } \\
\text { initial } \ldots \ldots \ldots \ldots \ldots \ldots\end{array}$ & $\begin{array}{l}0 \\
q\end{array}$ & $\begin{array}{l}7,50 \\
8,66\end{array}$ & $\begin{array}{l}6,25 \\
7,74\end{array}$ & $\begin{array}{c}\text { N.S. } \\
(\mathrm{P}<0,10) \\
\text { N.S. } \\
(\mathrm{P}<0,10)\end{array}$ & 11,74 & 11,96 & N.S \\
\hline $\begin{array}{l}\text { Température rectale } \\
\text { avant jeûne }\left({ }^{\circ} \mathrm{C}\right) \ldots .\end{array}$ & $\stackrel{0}{0}$ & $\begin{array}{l}41,6 \\
41,5\end{array}$ & $\begin{array}{l}41,5 \\
41,5\end{array}$ & $\begin{array}{l}\text { N.S. } \\
\text { N.S. }\end{array}$ & 40,8 & 40,2 & $* * *$ \\
\hline $\begin{array}{l}\text { Température rectale } \\
\text { en fin de jeûne }\left({ }^{\circ} \mathrm{C}\right)\end{array}$ & $\underset{q}{o}$ & $\begin{array}{l}40,5 \\
41,0\end{array}$ & $\begin{array}{l}40,4 \\
40,7\end{array}$ & $\begin{array}{l}\text { N.S. } \\
\text { N.S. }\end{array}$ & 40,3 & 39,8 & $* *$ \\
\hline $\begin{array}{l}\text { Glucose plasmatique } \\
\text { avant jeûne } \\
(\mathrm{mg} / 100 \mathrm{ml}) \ldots \ldots\end{array}$ & Q & & & & 232 & 225 & N.S. \\
\hline $\begin{array}{l}\text { Glucose plasmatique } \\
\text { en fin de jeûne } \\
(\mathrm{mg} / 100 \mathrm{ml}) \ldots \ldots \ldots\end{array}$ & O & & & & 218 & 209 & $* *$ \\
\hline $\begin{array}{l}\text { Acide urique plasma- } \\
\text { tique avant jeûne } \\
(\mathrm{mg} / \mathrm{l}) \ldots \ldots \ldots \ldots \ldots\end{array}$ & Q & & & & 34,9 & 41,6 & $* *$ \\
\hline $\begin{array}{l}\text { Acide urique plasma- } \\
\text { tique en fin de jeûne } \\
(\mathrm{mg} / \mathrm{l}) \ldots \ldots \ldots \ldots \ldots\end{array}$ & Q & & & & 30,8 & 39,5 & $* *$ \\
\hline
\end{tabular}

******: différence entre génotypes significative respectivement au seuil 5,1 et $0,1 \%$.

La baisse de température rectale en présence du gène $\mathrm{dw}$, associée à un métabolisme basal légèrement réduit, a déjà été signalée (cf. références citées dans GuILLAUME, 1976) ; elle n'est significative qu'en cages individuelles, tant avant la privation d'aliment qu'en fin de jeûne. Au sol, une différence de même sens n'est suggérée, sans être significative, 
TABLEAU 2

Animaux au sol : analyse de variance pour la perte de poids (en \%) pendant le jeûne. Birds in floor pens : variance analysis for body weight loss (per cent) during starvation

\begin{tabular}{|c|c|c|}
\hline Source de variation & $\begin{array}{l}\text { Degrés de } \\
\text { liberté }\end{array}$ & Valeur de $F$ et signification \\
\hline Génotype ........ & 1 & $4,53 *$ \\
\hline Sexe $\ldots \ldots \ldots \ldots$ & 1 & $6,77^{*}$ \\
\hline Interaction $\ldots \ldots \ldots \ldots \ldots \ldots$ & 1 & 0,10 \\
\hline Résiduelle & 122 & 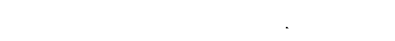 \\
\hline
\end{tabular}

- * : significatif au seuil $5 \%$.

- valeur de la variance résiduelle $=7,37$

qu'après effet du jeûne. Indépendamment du génotype, l'effet dépressif du jeûne sur la température interne apparaît dans tous les cas, conformément aux observations antérieures (cf. BIERER et al., 1965 ; STURKIE 1976). Il semble que cette même température soit systématiquement moins élevée (de $3 / 4$ à plus de $1^{\circ} \mathrm{C}$ ) en cages qu'au sol. La température ambiante étant la même, ceci doit être expliqué par d'autres facteurs (activité ; possibilité ou non de groupement des animaux ?...).

Le taux plasmatique de glucose, mesuré chez les poulettes en cages, est significativement plus bas pour les naines (dw) que pour les normales en fin de jeûne. La différence, non significative, est de même sens avant jeûne. Ces observations s'accordent avec la suggestion de GuILlAUME (1972) et avec les résultats de BANERJEE et al., (1981) sur des poulettes de 16 semaines. Pour le taux d'acide urique du plasma, avant jeûne et en fin de jeûne, il est plus élevé, de façon hautement significative, pour les poulettes naines que pour les normales. Ici encore, cette observation rejoint celle de BANERJEE et al., (1981) sur des poulettes Leghorn blanches normales et naines, âgées de 16 semaines. La différence entre génotypes est moins considérable dans le cas présent, mais il s'agit d'animaux plus jeunes et d'origine génétique différente. Enfin, l'effet du jeûne sur les deux paramètres sanguins, glucose et acide urique, apparaît clairement.

\section{B. Reprise de poids et indice de consommation après jeûne}

En cages individuelles, le gain de poids relatif (\% du poids initial) est légèrement inférieur pour les femelles dw comparées aux $\mathrm{Dw}^{+}, \operatorname{tant}$ de 8 à 9 que de 9 à 10 semaines. Au sol, de 8 à 9 semaines, les oiseaux " nains " des deux sexes ayant jeûné regagnent relativement moins de poids que les normaux. Par contre, la semaine suivante, tous les gains relatifs sont plus faibles, mais ils sont d'environ $2,5 \%$ plus élevés chez les poulets " nains " que chez les normaux. Si l'on compare par semaine et par génotype la différence des gains de poids relatifs en lot traité et en lot témoin, on conclut que, outre le " rattrapage " de poids général pour le lot traité, les « nains » ne sont pas incapables d'une certaine croissance compensatrice, mais celle-ci se manifeste avec retard par rapport au génotype normal. Peut-être le caractère répété et relativement rapproché des jeûnes réalisés par SIMON (1972) ne permettait-il pas cette compensation tardive. 
(⿸丆口

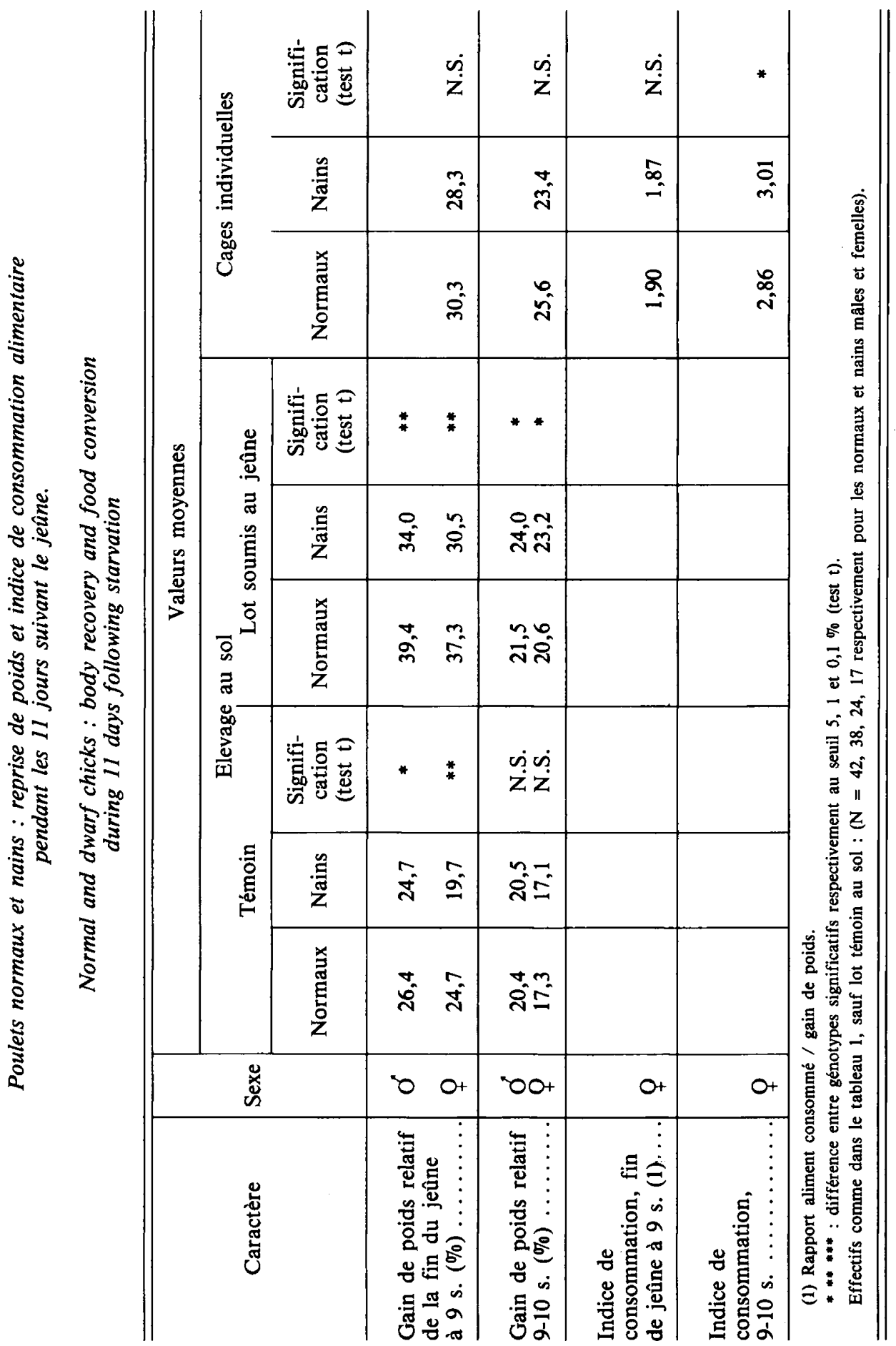




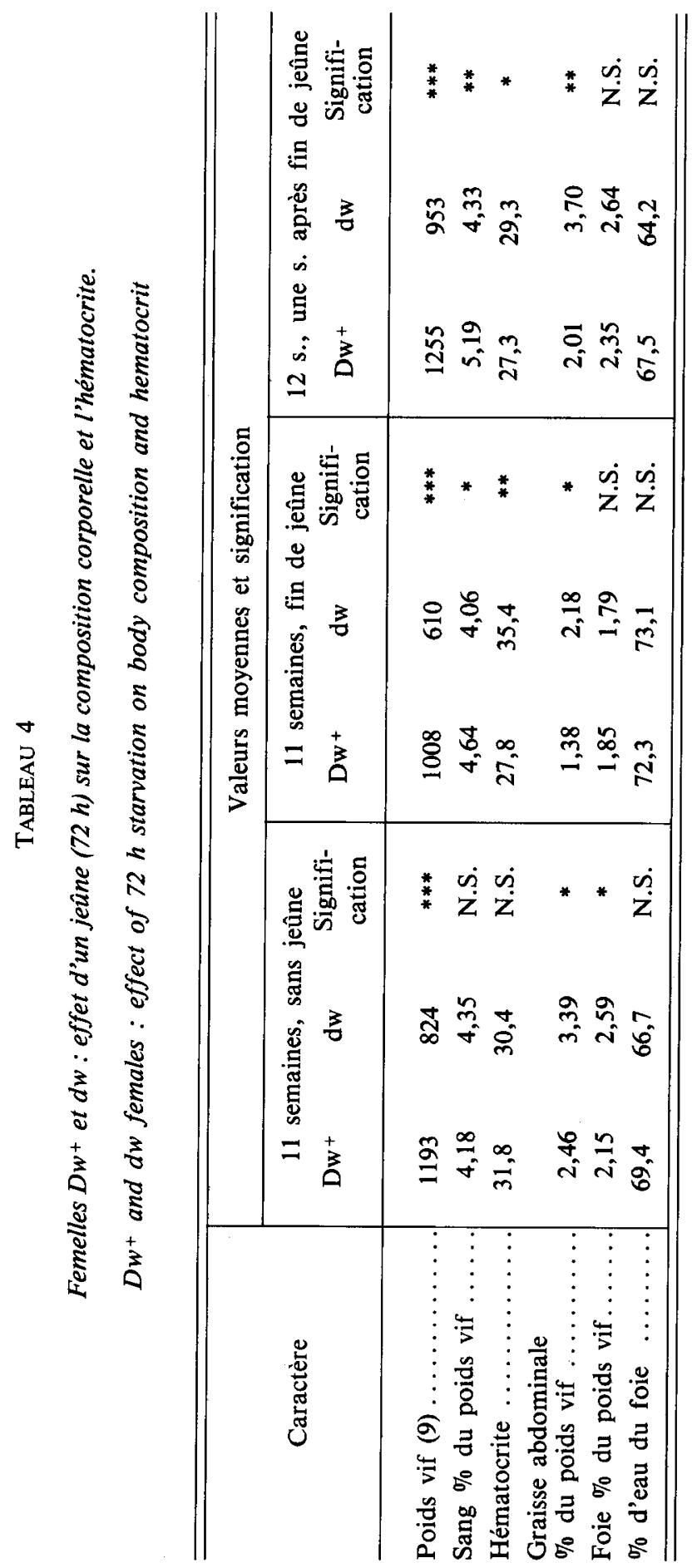


Les indices de consommation, mesurés en cages, apparaissent spécialement faibles lors des 4 premiers jours de réalimentation. Ils ne sont alors pas significativement différents pour les génotypes $\mathrm{Dw}^{+}$et $\mathrm{dw}$, contrairement au cas habituel.

\section{Composition corporelle et hématocrite}

Au tableau 4 on retrouve, pour la graisse abdominale en $\%$ du poids corporel, le fait connu (p. ex. Guillaume, 1976) qu'en alimentation ad libitum sa valeur moyenne est plus élevée pour les poulettes $d w$ que pour les $\mathrm{Dw}^{+}$. Le jeûne abaisse cette valeur de la même manière pour les deux génotypes, puis, une semaine après, la remontée est particulièrement marquée pour le génotype nain qui est le seul à dépasser la valeur initiale.

Le poids du foie (en $\%$ du poids vif), devient, à la fin du jeûne, sensiblement égal pour les deux génotypes, alors qu'en l'absence de jeûne, il est, comme la graisse abdominale, supérieur chez les naines. Le pourcentage d'eau de cet organe (en relation inverse avec le pourcentage de lipides) suit des fluctuations inverses de son poids total et de la graisse abdominale. En résumé, il semble que le foie, qui diminue relativement plus en poids, perde davantage de lipides chez les femelles dw.

Le pourcentage de sang, déduit des pertes à la saignée et non différent entre génotypes au départ, devient plus faible pour les naines en fin de jeûne et le reste la semaine suivante. Inversement, l'hématocrite, non significativement différent en l'absence de jeûne, devient en fin de jeûne notablement plus élevé pour les femelles $d w$ que pour les $D w^{+}$, ayant augmenté chez les premières et baissé chez les secondes. Le rapprochement des variations des deux paramètres, $\%$ du sang estimé d'après les pertes à la saignée et hématocrite chez les poulettes naines, suggère de rechercher si la consommation de l'eau (apportée ad libitum) ne pourrait être spontanément restreinte chez les naines et non chez les normales pendant la suppression d'aliment. Rappelons que l'excrétion d'eau ne diffère pas entre génotypes.

Il n'existe pas, à notre connaissance, de comparaisons publiées concernant la réponse de génotypes à une privation d'aliment pour les paramètres ci-dessus. L'effet du jeûne luimême sur ces paramètres, par contre, est connu. Citons, pour la graisse abdominale, une revue des travaux par STURKIE (1976) ; pour le poids du foie, BRADY et al., (1978) ; pour le pourcentage d'eau de cet organe, BESCH et al., (1972). Pour l'hématocrite, il ne semble pas qu'un effet d'une privation de l'aliment seul ait été signalé. Dans les données présentes, on observe sa diminution pendant la semaine qui suit la fin du jeûne.

\section{Conclusion}

Au total, avec la durée du jeûne réalisé ici, des différences relativement limitées se révèlent entre les poulets nains et ceux de taille normale. Pour la perte de poids pendant le jeûne, les poulets nains seraient légèrement moins sensibles que les normaux, mais au sol seulement. La baisse de température, celle du glucose et de l'acide urique plasmatique sont peu différentes. La reprise de poids relative par la suite est dans l'ensemble inférieure pour les oiseaux $\mathrm{dw}$, mais elle est décalée dans le temps et un peu supérieure la seconde semaine au sol, et le rétablissement du pourcentage de lipides abdominaux est plus précoce pour ce génotype. Par contre, le poids du foie en période de jeûne diminue davantage pour les poulettes naines. Quant à l'augmentation de l'hématocrite des femelles dw dans la même période, elle reste à expliquer. 


\section{Références bibliographiques}

Banerjee A.K., Bordas A., Merat P., 1981. The effect of temperature and genotype on growth traits, plasma glucose and uric acid in Dwarf and Normal White Leghorn females. Ann. Génét. Sél. anim., 13, 255-268.

Bell D.J., Freeman B.M., 1971 (editors). Physiology and biochemistry of the domestic fowl. Academic Press, New York.

BesCh E.L., Burton A.R., Smith A.H., 1972. Organ and body mass changes in restrained and fasted domestic fowl. Pro. Soc. exp. Biol. Med. 141, 456-459.

Bierer B.W., Eleasar T.H., RoEbuCK D.Z., 1965. Effect of feed and water deprivation on chicks, turkeys and laboratory animals. Poult. Sci., 44, 768-773.

Brady L.J., Romsos D.R., BRAdy P.S., Leveille G.A., 1978. The effect of fasting on body composition, glucose turnover, enzymes and metabolites in chickens. $J$. Nutr. 108, 648-657.

Guillaume J., 1972. Quelques particularités nutritionnelles et physiologiques du poussin nain dw. Ann. Génét. Sél. anim., 4, 233-250.

Guillaume J., 1976. The dwarfing gene dw : its effects on anatomy, physiology, nutrition, mangement. Its application in poultry industry. World's Poult. Sci. J. 32, 285-304.

KAWAHARA T., 1962. Endurance of one day-old chicks under starvation. Nat. Ind. of Genetics annual report, 13, 37-38.

RICARD F.H., Rouvier R., 1967. Étude de la composition anatomique du poulet de chair. I - Variabilité de la répartition des différentes parties corporelles chez des coquelets Bresse Pile. Ann. Zootech. 16, 23-39.

Rombauts P., Bordas A., Banerjee A.K., Merat P., 1982. Taux de corticostérone plasmatique, variation de poids et de température de poules Leghorn blanches naines (dw) et normles (Dw) en réponse à une privation d'aliment. Génét. Sél. Evol. 15, 251-256.

Simon J., 1972. Influence du gène de nanisme $(\mathrm{dw})$, du gène cou nu $(\mathrm{Na})$ et du rythme d'alimentation sur la croissance et le comportement alimentaire du poulet. Ann. Génét. Sél. anim. 4, 305-310.

Sturkie P.D., 1976. Avian Physiology. 3e ed. Springer, Berlin.

Ueno T., Miyazono Y., Komiyama T., 1977 : (Breed differences in the pattern of physiological response in chicks to feed and / or water deprivation). Bull. nat. Inst. anim. Industry 32, 29-37.

WASHBURN K.W., BONDARI K., 1978. Comparison of the effects of starvation and duration of restricted feeding on growth feed consumption and feed conversion of broiler chicks grown in individual cages and floor pens. Archiv. Geflügelk., 42, 94-98;

Zein-El-Dein M., Merat P., Bordas A., 1982. Gène " cou nu » et réponse à un jeûne de 48 heures en phase de croissance. Ann. Génét. Sél. anim., 14, (4), 373-378. 